\title{
Clinical activity score as a guide in the management of patients with Graves' ophthalmopathy
}

\author{
Maarten Ph. Mourits, Mark F. Prummel*, \\ Wilmar M. Wiersinga* and L. Koornneef \\ Orbital Centre and *Department of Endocrinology, \\ Academic Medical Center, University of Amsterdam, \\ The Netherlands
}

(Received 10 September 1996; returned for revision 27 October 1996; finally revised 13 November 1996; accepted 25 April 1997)

\section{Summary}

OBJECTIVE Approximately $35 \%$ of patients with Graves' ophthalmopathy do not respond to immunosuppressive treatment. A possible explanation for this finding is that only patients with active ophthalmopathy respond to immunosuppressive treatment, whereas patients with fibrotic end stage disease do not. To distinguish between these two groups and to predict the outcome of immunosuppressive treatment, we developed a clinical activity score (CAS) based on four of the five classical signs of inflammation and tested its efficacy in a double-blind, prospective study.

DESIGN, PATIENTS AND MEASUREMENTS The CAS was determined by an ophthalmologist before, on the day of, and after the start of either oral prednisone or retrobulbar irradiation in $\mathbf{4 3}$ patients with moderate to severe Graves' ophthalmopathy. The therapeutic outcome was determined by a second ophthalmologist unaware of the CAS stores given. Success of treatment was defined as an improvement in NOSPECS class or grade.

RESULTS Responders (22) and non-responders (21) did not differ in age, sex, duration or severity of their Graves' ophthalmopathy. The pretreatment CAS, however, was significantly higher in responders than in non-responders. Twelve of 22 responders and three of 21 non-responders had a CAS $\geq 4(80 \%$ vs $36 \% ; P<0.01$ ). Using this CAS cut-off point, the accuracy of CAS in predicting the therapeutic outcome was: specificity $86 \%$, sensitivity $55 \%$, positive

Present address and correspondence: M. Ph. Mourits, Department of Ophthalmology, University Hospital Utrecht, Heidelberglaan 100, 3508 GA Utrecht, The Netherlands. Fax: 030254 1855. E-mail: M.P.Mourits@oogh.azu.nl. predictive value $80 \%$, negative predictive value $64 \%$. Patients with a CAS $\geq 4$ had a similar duration of Graves' ophthalmopathy as patients with a CAS $<4$. CONCLUSIONS The clinical activity score has a high predictive value for the outcome of immunosuppressive treatment in Graves' ophthalmopathy. Disease activity, and not disease duration, is the prime determinant of therapeutic outcome.

Graves' ophthalmopathy is a disease characterized by enlargement of the extraocular muscles and an increase in retrobulbar fat (Bahn \& Heufelder, 1993). As a consequence, the eyelids become swollen and the eyes proptotic. Orbital fibroblasts are thought to play a crucial role in the pathogenesis by their ability to produce hydrophilic glycosaminoglycans. The presence of activated $\mathrm{CD}^{+}$lymphocytes in the orbits of patients with Graves' ophthalmopathy and the interactions of these cells with retrobulbar fibroblasts together with other supporting findings has led to the assumption that Graves' ophthalmopathy is an organ-specific autoimmune disease and can therefore be treated by means that suppress the immune process (Burch \& Wartofsky, 1993). One such treatment is administration of corticosteroids either intravenously or orally. Another is retrobulbar external irradiation in a total dosage of $20 \mathrm{~Gy}$, which inhibits but does not kill the fibroblasts present (Kahaly \& Beyer, 1988). Several studies have shown that both treatment modalities proved effective in a majority of patients (Wiersinga et al., 1988; Prummel et al., 1989, 1993a). However, a substantial number did not respond to these treatments (Mourits et al., 1989). As Graves' ophthalmopathy appears to be biphasic, with an initial phase of active and progressive disease followed by a 'burnt out' phase in which alterations such as proptosis and motility restrictions remain stable, we hypothesized that immunosuppression would be beneficial in only the early, active phase of the disease. This assumption is generally accepted, but never proved. We, therefore, tried to develop an 'activity score' in patients with Graves' ophthalmopathy, that could predict which patients would benefit from immunosuppressive treatment and which would not. This clinical activity score (CAS) is based on four of the five well-known classical signs of inflammation (pain, redness, swelling and impaired function) and consists of 10 items (Table 1). For each item present, one point is given. Each item has the same weight. The sum of these points is the CAS (range $0-10$ ). In a retrospective pilot study we found a positive 
Table 1 The 10 items of the clinical activity score (CAS)

\begin{tabular}{|c|c|c|}
\hline \multirow[t]{2}{*}{ Pain } & 1 & $\begin{array}{l}\text { Painful, oppressive feeling on or behind the } \\
\text { globe, during the last } 4 \text { weeks }\end{array}$ \\
\hline & 2 & $\begin{array}{l}\text { Pain on attempted up, side or down gaze, } \\
\text { during the last } 4 \text { weeks }\end{array}$ \\
\hline \multirow[t]{2}{*}{ Redness } & 3 & Redness of the eyelid(s) \\
\hline & 4 & $\begin{array}{l}\text { Diffuse redness of the conjunctiva, covering } \\
\text { at least one quadrant }\end{array}$ \\
\hline \multirow[t]{4}{*}{ Swelling } & 5 & Swelling of the eyelid(s) \\
\hline & 6 & Chemosis \\
\hline & 7 & Swollen caruncle \\
\hline & 8 & $\begin{array}{l}\text { Increase of proptosis of } \geq 2 \mathrm{~mm} \text { during a } \\
\text { period of } 1-3 \text { months }\end{array}$ \\
\hline \multirow[t]{2}{*}{ Impaired function } & 9 & $\begin{array}{l}\text { Decrease of eye movements in any direction } \\
\geq 5^{\circ} \text { during a period of } 1-3 \text { months }\end{array}$ \\
\hline & 10 & $\begin{array}{l}\text { Decrease of visual acuity of } \geq 1 \text { line(s) on } \\
\text { the Snellen chart (using a pinhole) during } \\
\text { a period of } 1-3 \text { months }\end{array}$ \\
\hline
\end{tabular}

For each item present, 1 point is given. The sum of these points is the CAS, e.g. a CAS of 6 means taht six items were present, regardless of which items.

relationship between this CAS and the outcome of immunosuppressive treatment (Mourits et al., 1989). The present study was undertaken to test the usefulness of the CAS in a prospective, double-blind trial in which patients with moderate to severe Graves' ophthalmopathy were treated with either prednisone or with retrobulbar irradiation.

\section{Methods}

\section{Patients}

We studied patients with moderately severe Graves' ophthalmopathy, who were between 20 and 75 years old. All had given their informed consent for the study. All had been euthyroid for at least 3 months, as indicated by the presence of normal plasma concentrations of T4 and T3, and by TSH concentrations that were within or below the normal range (normal ranges: T4, $70-150 \mathrm{nmol} / \mathrm{l}$; T3, $1 \cdot 30-2 \cdot 45 \mathrm{nmol} / \mathrm{l}$; TSH, $0 \cdot 4-4 \cdot 0 \mathrm{mU} / \mathrm{l})$. Most patients were on antithyroid drugs in combination with $\mathrm{T} 4$ supplementation $(n=28)$. Five patients used T4 after previous ablative treatment, six were in remission of previous Graves' hyperthyroidism, while in four patients no evidence for Graves' thyroid disease was found. The diagnosis of Graves' ophthalmopathy was based on the presence of the typical clinical features in combination with enlarged extraocular eye muscles on a coronal CT scan. We included only patients with moderately severe ophthalmopathy, defined as having one or more of the following NOSPECS categories: class 2, grade c; class 3 , grades abc; class 4 , grades abc and class 6 , grade a
Table 2 The NOSPECS classification of ocular changes in Graves' ophthalmopathy*

\begin{tabular}{|c|c|c|}
\hline Class & Grade & Change \\
\hline 0 & & No signs or symptoms \\
\hline 1 & & Only signs \\
\hline 2 & $\begin{array}{l}\mathrm{o} \\
\mathrm{a} \\
\mathrm{b} \\
\mathrm{c}\end{array}$ & $\begin{array}{l}\text { Soft tissue involvement, with symptoms and signs } \\
\text { absent } \\
\text { minimal } \\
\text { moderate } \\
\text { marked }\end{array}$ \\
\hline 3 & $\begin{array}{l}\mathrm{o} \\
\mathrm{a} \\
\mathrm{b} \\
\mathrm{c}\end{array}$ & $\begin{array}{l}\text { Proptosis } \\
\qquad 23 \mathrm{~mm} \\
23-24 \mathrm{~mm} \\
25-27 \mathrm{~mm} \\
\geq 28 \mathrm{~mm}\end{array}$ \\
\hline 4 & $\begin{array}{l}\mathrm{o} \\
\mathrm{a} \\
\mathrm{b} \\
\mathrm{c}\end{array}$ & $\begin{array}{l}\text { Extraocular muscle involvement } \\
\text { absent } \\
\text { limitation of motion in extremes of gaze } \\
\text { evident restriction of motion } \\
\text { fixation of a globe or globes }\end{array}$ \\
\hline 5 & $\begin{array}{l}\mathrm{o} \\
\mathrm{a} \\
\mathrm{b} \\
\mathrm{c}\end{array}$ & $\begin{array}{l}\text { Corneal involvement } \\
\text { absent } \\
\text { stippling of cornea } \\
\text { ulceration } \\
\text { clouding, necrosis, perforation }\end{array}$ \\
\hline 6 & $\begin{array}{l}\mathrm{o} \\
\mathrm{a} \\
\mathrm{b} \\
\mathrm{c}\end{array}$ & $\begin{array}{l}\text { Sight loss (visual acuity } \dagger \text { ) } \\
\quad>0 \cdot 67 \\
0 \cdot 67-0 \cdot 33 \\
0 \cdot 33-0 \cdot 10 \\
<0 \cdot 10\end{array}$ \\
\hline
\end{tabular}

* Modified slightly from the classification of Werner (1977).

$\dagger$ Expressed as a decimal, e.g. $20 / 20=1 \cdot 0,20 / 30=0 \cdot 67,20 / 60=$ $0 \cdot 33,20 / 200=0 \cdot 10$.

(Table 2; Werner, 1977). Patients with diabetes mellitus were not included, neither were patients with contraindications for corticosteroids or external beam irradiation. Patients with rapidly decreasing visual functions or other signs of optic nerve compression by apical crowding were not included. Prior treatment other than eyedrops, gels or ointments excluded patients from participation. Patients with insufficient follow-up, or who developed abnormal thyroid function during the study were excluded from the analysis and replaced. The study was approved by the Medical Ethics Committee of the University of Amsterdam.

\section{Treatment}

The patients participated in a clinical trial comparing oral prednisone and radiotherapy (Prummel et al., 1993a), and were randomly assigned to receive oral prednisone or retrobulbar external beam irradiation. Prednisone was given in decreasing 
doses: $60 \mathrm{mg}$ for the first 2 weeks, $40 \mathrm{mg}$ for 2 weeks, $30 \mathrm{mg}$ for 4 weeks and $20 \mathrm{mg}$ for 4 weeks; after that the dose was tapered off by $2.5 \mathrm{mg}$ per week. Retrobulbar radiotherapy was administered with a $5 \mathrm{meV}$ linear accelerator in 10 divided fractions of $2 \mathrm{~Gy}$ daily over a 2 week period. Localization and verification films with lead markers on the canthus of each eye were performed for each patient on a simulator (Donaldson et al., 1973). The dose was calculated at the midline and administered by two $3^{\circ}$ posterior angled lateral portals of $5 \times 5 \mathrm{~cm}$, with the patient's head fixed by a full head shell. Thyroid medication was not changed during the study period.

\section{Assessment of the CAS, NOSPECS classification and therapeutic outcome}

To assess the disease activity, the CAS was used (Table 1). To assess the disease severity and the outcome of treatment, the NOSPECS classification was used (Table 2).

The CAS was determined in all patients 2 days before $\left(\mathrm{T}^{-2}\right)$, on the day $\left(\mathrm{T}^{0}\right)$ of the start of treatment and 24 weeks $\left(\mathrm{T}^{24}\right)$ later by an ophthalmologist (L.K.) who was otherwise not involved in the diagnosis or treatment of these patients. The CAS on $\mathrm{T}^{0}$ was used to establish the relationship with the outcome of treatment. The CAS on $\mathrm{T}^{-2}$ and on $\mathrm{T}^{0}$ were used to calculate the Kappa value, thus determining the intraobserver agreement. The changes between the CAS on $\mathrm{T}^{0}$ and $\mathrm{T}^{24}$ served to assess the effect of immunosuppressive treatment on the disease activity.

Patients were classified according to the NOSPECS classification by a second ophthalmologist (M.P.M.) on the day of the start of treatment and 4, 12 and 24 weeks later. This second ophthalmologist has no knowledge of the CAS that had been given to the patients. Proptosis was measured with the same Hertel exophthalmometer, and pinhole visual acuity was tested with the Snellen chart and expressed as a decimal (e.g. $20 / 20=1 \cdot 0 ; 20 / 30=0 \cdot 67 ; 20 / 60=0 \cdot 33$ ). The ocular motility was described in terms of the NOSPECS classification (e.g. normal, slightly, moderately and severely impaired). In addition, the eye movements in four directions (up, down and sideways) were measured in degrees by an orthoptic assistant using a Maddox cross (Crone, 1973). As the end-point of the study we determined the therapeutic outcome at 24 weeks after start of treatment. A response was defined as a decrease in NOSPECS class or, if this did not occur, in grade from the baseline value. A lack of response was indicated by an absence of change in class or grade, or by treatment failure if an increase in class or grade occurred. If both increases an decreases were observed, the change in the highest NOSPECS class determined the therapeutic outcome (Prummel et al., 1989). Because corneal involvement in Graves' ophthalmopathy usually disappears with local measures and therefore does not necessarily indicate more severe eye disease, NOSPECS class 5 was not used to determine treatment response.

\section{Statistical analysis}

The results were analysed with the SYSTAT (Systat Inc., Evanston, IL, USA) software package. All values are expressed as the mean $\pm \mathrm{SD}$ of values recorded for affected orbits. $P$ values of less than 0.05 were considered to indicate significance. To compare percentages we used the $\chi^{2}$ test; changes in ophthalmological characteristics and in biochemical variables were analysed by analysis of variance using the repeated measures $F$-test. Intraobserver agreement of CAS on $\mathrm{T}^{-2}$ and $\mathrm{T}^{0}$ was determined by calculating the Kappa values. Kappa is defined as the actual agreement beyond chance divided by the potential agreement beyond chance (Fleiss, 1981).

\section{Results}

The study group consisted of 43 patients who fulfilled the inclusion criteria. Twenty-two patients were treated with prednisone and 21 received radiotherapy. One patient developed a recurrence of hyperthyroidism during the study period and was replaced. Two patients showed a serious deterioration of their ophthalmopathy at the end of the study period and did not complete the follow-up of 24 weeks. The CAS at the time of their premature withdrawal, however, was determined and their data were included in the analysis.

The therapeutic outcome after 24 weeks, as determined by the change in the highest NOSPECS class, did not differ in the prednisone group (10 of 22 responders) and the radiotherapy group (12 of 21 responders). The total success rate was $51 \%$. Responders and non-responders did not differ in age $(43.5 \pm 11 \cdot 9$ vs $46 \cdot 2 \pm 11 \cdot 4$ years (mean $\pm \mathrm{SD}$ ), not significant) or sex (20 females/2 males vs 17 females/4 males, not significant). The duration of their ophthalmopathy was similar (median 14 (range 6-120) vs median 12 (range 5-96) months, not significant). Also, the severity as expressed as the Total Eye Score (TES $=$ sum of each NOSPECS class present multiplied by the grade within that class, in which we substituted a, b and c, respectively for 1,2 and 3$)$ was not different $(10 \cdot 8 \pm 4 \cdot 7 \mathrm{vs}$ $9 \cdot 8 \pm 3 \cdot 7$ (mean $\pm \mathrm{SD}$ ), not significant). However, the disease activity as indicated by the CAS on $\mathrm{T}^{0}$ was significantly higher in responders than in non-responders $(3 \cdot 4 \pm 2 \cdot 0$ vs $2 \cdot 4 \pm 1 \cdot 2$ (mean $\pm \mathrm{SD}), P=0 \cdot 05$ ).

The relationship between the CAS on $\mathrm{T}^{0}$ and the therapeutic outcome is depicted in Fig. 1. Twelve of 22 responders and three of 21 non-responders had a CAS $\geq 4(80 \%$ vs 36\%; $P<0 \cdot 01$ ). Taking this CAS cut-off point, the accuracy of CAS in predicting the therapeutic outcome is given in Table 3). The accuracies of the individual items of the CAS are given 


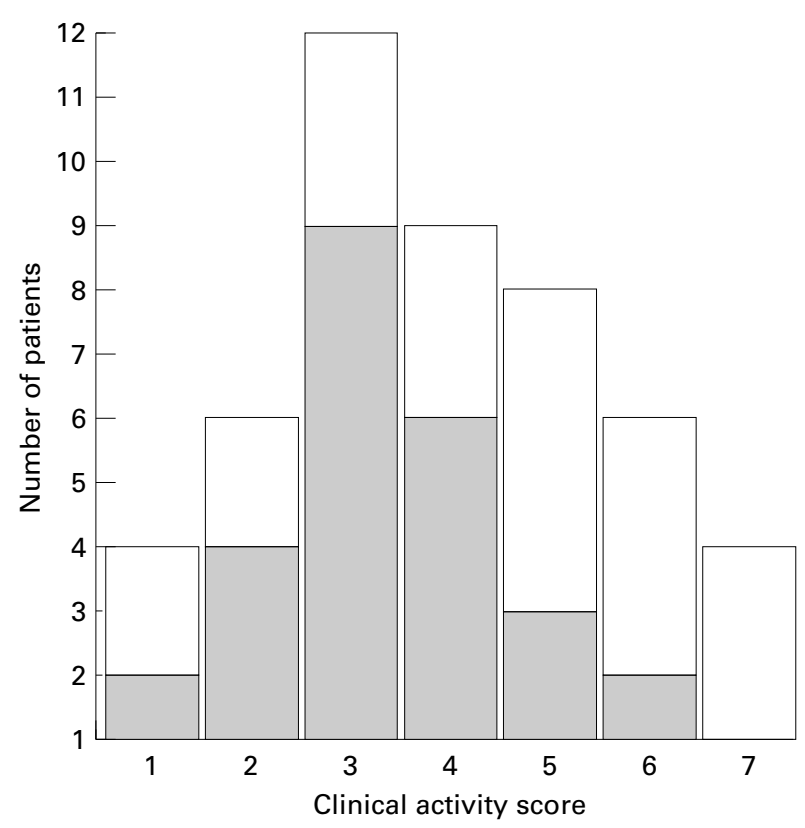

Fig. 1 Diagram showing the relationship between CAS and therapeutic outcome. $\square$ success, $\square$ no success.

in Table 4. If a CAS cut-off point of 3 was used, this resulted in less acceptable values (positive predictive value $61 \%$, negative predictive value $60 \%$, sensitivity $64 \%$ and specificity $57 \%$ ). Comparing patients with a CAS $\geqslant 4$ and patients with a CAS $<4$, we found a similar duration of Graves' ophthalmopathy (median 17.0 (range 7-120) vs median 12.0 (range 5-96) months, not significant (Table 5)), but a different severity of the ophthalmopathy in both groups $(10.9 \pm 4.5$ vs $8 \cdot 1 \pm 2 \cdot 9$ (mean $\pm \mathrm{SD}), \quad P=0 \cdot 001$ ). Twenty-four weeks after the start of treatment, CAS had decreased only in responders ( $3.4 \pm 2.0$ to $1.9 \pm 1.4$ (mean $\pm \mathrm{SD}$ ), $P<0.001$ ), whereas it did not change in non-responders $(2 \cdot 4 \pm 1 \cdot 2$ to $2 \cdot 7 \pm 1 \cdot 9$ (mean $\pm \mathrm{SD}$ ), not significant).

The reproducibility of the pretreatment individual CAS items

Table 3 Accuracy of clinical activity score (CAS) in predicting therapeutic outcome of immunosuppression in Graves' ophthalmopathy

\begin{tabular}{lccc}
\hline & Success & No success & \\
\hline CAS $\geq 4$ & 12 & 3 & 15 \\
CAS $<4$ & 10 & 18 & 28 \\
Total & 22 & 21 & 43 \\
\hline
\end{tabular}

Positive predictive value, 12 of $15=80 \%$; negative predictive value, 18 of $28=64 \%$; sensitivity, 12 of $22=55 \%$; specificity, 18 of $21=86 \%$.
Table 4 Observed agreement and predictive value of individual items of the clinical activity score (CAS)

\begin{tabular}{|c|c|c|c|c|c|c|c|c|}
\hline \multirow[b]{2}{*}{ CAS item } & \multicolumn{2}{|c|}{ Incidence } & \multirow[b]{2}{*}{ OA } & \multirow[b]{2}{*}{$\mathrm{Ka}$} & \multirow[b]{2}{*}{ PPV } & \multirow[b]{2}{*}{ NPV } & \multirow[b]{2}{*}{ Sens } & \multirow[b]{2}{*}{ Spec } \\
\hline & $\mathrm{T}^{0}$ & $\mathrm{~T}^{-2}$ & & & & & & \\
\hline Pain at rest & 53 & 60 & 84 & 67 & 57 & 59 & 57 & 57 \\
\hline Pain moving & 49 & 49 & 86 & 72 & 50 & 52 & 52 & 52 \\
\hline Red eyelid & 23 & 26 & 84 & 57 & 60 & 52 & 27 & 81 \\
\hline Red conjunctiva & 28 & 19 & 77 & 36 & 67 & 55 & 36 & 81 \\
\hline Swelling lid & 91 & 74 & 84 & 47 & 51 & 50 & 91 & 10 \\
\hline Chemosis & 21 & 26 & 84 & 57 & 78 & 56 & 47 & 90 \\
\hline Caruncle & 2 & 2 & 100 & 100 & 100 & 50 & 5 & 100 \\
\hline Proptosis & 19 & 19 & 100 & 100 & 100 & 58 & 32 & 100 \\
\hline Motility & 7 & 7 & 100 & 100 & 100 & 53 & 14 & 100 \\
\hline Visual acuity & 2 & 2 & 100 & 100 & 100 & 50 & 5 & 100 \\
\hline Total CAS & - & - & 88 & 73 & 80 & 64 & 55 & 86 \\
\hline
\end{tabular}

Incidence, In per cent (on day of start of treatment, $\mathrm{T}^{0}$, and 2 days before, $\mathrm{T}^{-2}$ ); OA, observed agreement $\mathrm{T}^{0} / \mathrm{T}^{-2} ; \mathrm{Ka}$, Kappa value; PPV, positive predictive value; NPV, negative predictive value, Sens, sensitivity and Spec, specificity of the 10 items of the CAS and of the total CAS (cut-off point 4) in 43 patients treated for Graves' ophthalmopathy. For the calculation of the PPVs, NPVs, sensitivity and specificity the CAS items on $\mathrm{T}^{0}$ were used. PPVs, etc. were calculated for treatment outcomes expressed in NOSPECS score alteration.

as well of the total CAS (as determined on $\mathrm{T}^{-2}$ and on $\mathrm{T}^{0}$ by calculating the Kappa values) is shown in Table 4. Kappa values varied from 36 (chemosis) to 100 (caruncle swelling, proptosis increase, decrease in motility, decrease in visual acuity). Eyelid swelling was the most prevalent item, whereas caruncle swelling and decreased visual acuity were only observed once. Eyelid swelling appeared to have the lowest predictive value; caruncle swelling, increased proptosis, decreased motility and decreased visual acuity had the highest predictive values.

\section{Discussion}

Rundle (1964) has shown that the typical course of Graves' ophthalmopathy is characterized by an initial phase of rapid progression, reaching its peak after 6-24 months, followed by a plateau phase of varying duration, and then a gradual, often incomplete regression of symptoms. It is conceivable that the signs and symptoms of the initial phase reflect the inflammatory changes induced by the autoimmune process, whereas the white proptotic eyes with upper and lower lid retraction and unchanging motility impairment are expressions of the fibrotic end stage of the disease (Burch \& Wartofsky, 1993). As the duration of the various stages of the disease may vary 
Table 5 Comparison of baseline clinical and laboratory variables in 43 patients with Graves' ophthalmopathy divided in having a clinical activity score (CAS) $<4$ and CAS $\geq 4$

\begin{tabular}{lccc}
\hline & CAS $<4$ & CAS $\geq 4$ & $P$ value \\
\hline Number & 28 & 15 & \\
Male/female & $4 / 24$ & $3 / 12$ & NS \\
Age (years) & $44 \cdot 9 \pm 11 \cdot 7$ & $43 \cdot 3 \pm 12 \cdot 8$ & NS \\
Duration GO (months) & $12 \cdot 0(5-96)$ & $17 \cdot 0(7-120)$ & NS \\
Duration GTD (months) & $26 \cdot 0(6-96)$ & $24 \cdot 0(7-120)$ & NS \\
Severity GO (TES*) & $8 \cdot 1 \pm 2 \cdot 9$ & $10 \cdot 9 \pm 4 \cdot 5$ & $0 \cdot 001$ \\
Proptosis & $18 \cdot 6 \pm 3 \cdot 2$ & $21 \cdot 0 \pm 4 \cdot 0$ & $0 \cdot 003$ \\
Visual acuity & $0 \cdot 93 \pm 0 \cdot 1$ & $0 \cdot 88 \pm 0 \cdot 1$ & NS \\
T4 (nmol/l) & $126 \pm 31$ & $121 \pm 31$ & NS \\
FTI & $132 \pm 41$ & $123 \pm 27$ & NS \\
T3 (nmol/l) & $1 \cdot 90 \pm 0 \cdot 24$ & $1 \cdot 92 \pm 0 \cdot 27$ & NS \\
TSH (mU/l) & $0 \cdot 8 \pm 1 \cdot 0$ & $1 \cdot 4 \pm 1 \cdot 8$ & NS \\
& & & \\
\hline
\end{tabular}

* The total eye score (TES) is defined as the sum of each NOSPECS class present multiplied by the grade within that class (for this purpose we substituted a, b and c, respectively for 1,2 and 3). GO, Graves' ophthalmopathy; GTD, Graves' thyroid disease. Plasma concentrations of T4 and T3 were measured by conventional radioimmunoassays, and the free T4 index (FTI) was calculated using the T3 resin uptake. TSH was determined by immunoradiometric assay (Boots-Celltech Diagnostics Ltd, Slough, UK). Pretreatment values of thyroid function tests are given as mean \pm SD. considerably, the total duration of the ophthalmopathy itself is not an ideal determinant for staging. In previous studies and again in this study we could demonstrate that the outcome of treatment was not related to the total duration of the ophthalmopathy (Prummel et al., 1989, 1993a). Signs that reflect the inflammatory changes, such as vasodilatation, oedema, congestion and volume increase, seem more appropriate to use as clinical markers of disease activity. Since the times of Galen (AD 131-201), the acute inflammatory process has been described in terms of pain, redness, swelling, heat and impaired function. The CAS is based on these principal signs of inflammation. The item 'heat' was not incorporated, as detection of subtle changes in temperature is clinically impossible without special instruments.

This prospective, double-blind study confirmed the provisional conclusions of our retrospective pilot study: CAS has a high specificity and high positive predictive value in predicting the therapeutic outcome of immunosuppressive treatment and radiotherapy. Patients with a CAS $\geq 4$ had a $80 \%$ chance of a favourable treatment effect, whereas the $a$ priori chance in this study was $51 \%$. The intraobserver variance (expressed in Kappa values) appeared very reasonable. Subjective complaints, such as pain at rest and pain during eye movements, were reasonably consistently revealed. Redness of the conjunctiva had the lowest Kappa value, suggesting that this sign is difficult to recognize. It should be emphasized that redness of the conjunctiva as a result of corneal stippling or ulceration is not what we consider a sign of active inflammation of the orbital tissues, nor the dilated vessels in the lateral canthus over the extraocular muscle insertions. Redness of the conjunctiva as a sign of acute inflammation is a diffuse redness, covering at least one quadrant. The low Kappa values of the sign of redness may further be due to the biological variation. Swelling of the eyelids was diagnosed in almost all patients and was therefore non-specific. It was difficult to discriminate between eyelid swelling caused by oedema and by fat protrusion. However, only the former is thought to be related to the acute inflammatory process. Chemosis, caruncle swelling, increase in proptosis, decrease in motility and visual acuity, although present only in a small minority of patients, were all highly specific for disease activity and therapeutic outcome.

We tried to correlate the CAS without the three last items with the therapeutic outcome, but found no relationship. Therefore, two visits with at least a month in between, are required to assess the CAS. In practice, we do not consider this a major disadvantage as this time can be used to collect data on the patient's status (thyroid function, CT scan, etc.). More important is the fact that, although very specific, the CAS is not very sensitive. Thirty-six per cent of patients with a CAS $<4$ nevertheless showed a favourable outcome on treatment. We have tried to modify the CAS in such a way that some items had a double or triple weight, but this did not result in a more sensitive CAS. Therefore, we believe that the CAS should be used in combination with other parameters of disease activity.

Several laboratory parameters of disease activity in Graves' ophthalmopathy have been proposed. Kahaly has extensively studied the concentrations of glycosaminoglycans (GAGs) in the urine and plasma of patients with active and inactive, treated and not (yet) treated Graves' ophthalmopathy (Kahaly et al., 1990, 1994; Schuler et al., 1993). He found a significant 
increase in GAGs in both the urine and plasma of patients with Graves' ophthalmopathy as compared with control groups. This increase was most pronounced in patients described as having active disease (based on our CAS) and subsided after immunosuppressive treatment and radiotherapy. The concentrations of GAGs might therefore be used as a marker of disease activity. These are, however, influenced by several pathological conditions and their significance as a predictor of therapeutic outcome has still to be investigated. Hoh et al. (1994) used the short tau inversion recovery sequence in magnetic resonance imaging to estimate the water content (oedema) of orbital tissues, thus assessing disease activity in Graves' ophthalmopathy. They found a correlation between high signal intensity ratios and active disease as described in terms of our CAS. The predictability of therapeutic response using this method is currently being investigated. Prummel et al. (1993b) used A-mode ultrasonography to assess disease activity in Graves' ophthalmopathy and found a positive predictive value of $73 \%$ in predicting the outcome of prednisone treatment or radiotherapy, albeit in a very limited number of patients. Also, serum soluble interleukin-2 receptor and serum soluble CD8 concentrations have been proposed, but not tested, as markers of disease activity (Weryha et al., 1991; Balazs et al., 1994).

The advantage of the clinical activity score is that it is easy, cheap and entirely clinical. In patients in whom spontaneous recovery cannot be awaited, treatment usually consists of corticosteroid treatment, orbital irradiation or surgery. Total duration of the ophthalmopathy is not a good guide in deciding which therapy is most appropriate. Early surgery may result in recurrence of symptoms; immunosuppression by steroids or irradiation given in the fibrotic end stage may unnecessarily prolong the disease period. A high clinical activity score helps to select patients who will benefit from immunosuppressive treatment. A low clinical activity score, however, does not exclude favourable therapeutic results. Further studies to improve the sensitivity of the clinical activity score or the predictability or a combination of disease activity markers are therefore required.

\section{References}

Bahn, R.S. \& Heufelder, A.E. (1993) Pathogenesis of Graves' ophthalmopathy. New England Journal of Medicine, 329, 1468-1475.

Balazs, C., Bokk, A. \& Farid, N.R. (1994) Serum soluble CD8 concentrations is an indicator of disease activity in patients with Graves' disease. Thyroid, 4, 27-30.

Burch, H.B. \& Wartofsky, L. (1993) Graves' ophthalmopathy: Current concepts regarding pathogenesis and management. Endocrine Reviews, 14, 747-793.
Crone, R.A. (1973) Motility disturbances in cases of endocrine exophthalmos. In Diplopia (ed. R.A. Crone), pp. 350-360. Excerpta Medica, Amsterdam.

Donaldson, S.S., Bagshaw, M.A. \& Kriss, J.P. (1973) Supervoltage orbital radiotherapy for Graves' ophthalmopathy. Journal of Clinical Endocrinology \& Metabolism, 37, 276-285.

Fleiss, J.L. (1981) The measurement of interrater agreement. In Statistical Methods for Rates and Proportions, 2nd ed., pp. 212-235. John Wiley \& Sons, New York.

Hoh, H.B., Laitt, R.D., Wakeley, C., Kabala, J., Goddard, P., Potts, M.J. \& Harrad, R.A. (1994) The STIR sequence MRI in the assessments of extraocular muscles in thyroid eye disease. Eye, $\mathbf{8}$, 506-510.

Kahaly, G. \& Beyer, J. (1988) Immunosuppressant therapy of thyroid eye disease. Klinische Wochenschrifte, 66, 1049-1059.

Kahaly, G., Schuler, M., Sewel, A.C., Bernard, G., Beyer, J. \& Krause, U. (1990) Urinary glycosaminoglycans in Graves' ophthalmopathy. Clinical Endocrinology, 33, 35-44.

Kahaly, G., Hansen, C., Beyer, J. \& Winand, R. (1994) Plasma glycosaminoglycans in endocrine ophthalmopathy. Journal of Endocrinological Investigation, 17, 45-50.

Mourits, M.P., Koorneef, L., Wiersinga, W.M., Prummel, M.F., Berghout, A. \& Van der Gaag, R. (1989) Clinical criteria for the assessment of disease activity in Graves' ophthalmopathy: A novel approach. British Journal of Ophthalmology, 73, 639-644.

Prummel, M.F., Mourits, M.P., Berghout, A., Krenning, E.P., Van der Gaag, R., Koorneef, L. \& Wiersinga, W.M. (1989) Prednisone and cyclosporine in the treatment of severe Graves' ophthalmopathy. New England Journal of Medicine, 321, 1353-1359.

Prummel, M.F., Mourits, M.P., Blank, L., Koornneef, L. \& Wiersinga, W.M. (1993a) Randomised double-blind trial of prednisone versus radiotherapy in Graves' ophthalmopathy. Lancet, 342, 949-954.

Prummel, M.F., Suttorp-Schulten, M.S.A., Wiersinga, W.M., Verbeek, A.M., Mourits, M.P. \& Koornneef, L. (1993b) A new ultrasonographic method to detect disease activity and predict response to immunosuppressive treatment in Graves' ophthalmopathy. Ophthalmology, 100, 556-561.

Rundle, F.F. (1964) Eye signs of Graves' disease. In The Thyroid (ed. R. Pitt-Rivers \& W.R. Trotter), pp. 171-197. Butterworths and Co., Washington DC.

Schuler, M., Hansen, C., Winand, R., Stover, C., Otto, E., Beyer, J. \& Kahaly, G. (1993) Urinary and plasma glycosaminoglycans in endocrine ophthalmopathy. In Endocrine Ophthalmopathy. Molecular, Immunological and Clinical Aspects (ed. G. Kahaly), pp. 58-67. Karger Dev Ophthalmologie, Basel.

Werner, S.C. (1977) Modification of the classification of the eye changes of Graves' disease: Recommendations of the Ad Hoc Committee of the American Thyroid Association. Journal of Clinical Endocrinology \& Metabolism, 44, 203-204.

Weryha, G., Gobert, B., Leclere, J., Bene, M.C., Faure, G. \& Hartemann, P. (1991) Dynamic changes in soluble interleukine-2 receptor levels during treatment of Graves' disease. Correlation with disease activity. Hormonal Research, 35, 8-12.

Wiersinga, W.M., Smit, T., Schuster-Uittenhoeve, A.L., Van der Gaar, R. \& Koornneef, L. (1988) Therapeutic outcome of prednisone medication and of orbital irradiation in patients with Graves' ophthalmopathy. Ophthalmologica, 197, 75-84. 